\title{
A STUDY OF THE USE OF LASER SPECKLE TO MEASURE SMALL TILTS OF OPTICALLY ROUGH SURFACES ACCURATELY
}

\author{
H.J. TIZIANI \\ Institute of Applied Physics of the Swiss Federal Institute of Technology, (ETH), \\ 8049 Zurich, Switzerland
}

Received 10 April 1972

\begin{abstract}
A new method is proposed where small tilts can be measured with high accuracy even in the presence of a lateral shift. For this purpose the speckling of the object, an optically rough surface, is recorded in the Fourier plane before and after a tilt. By illuminating the developed plate with a laser, fringes with spacings inversely proportional to the tilt angle can be observed.
\end{abstract}

\section{Introduction}

The holographic techniques to study small threedimensional movements were analysed extensively by many scientists the last few years and a few references will be given only [1-4]. Methods of applying speckling for the measurement of in-plane translations were also investigated recently by a number of authors [5-13].

In the present work, speckling is applied to mea: sure tilts occurring in the presence of other movements, very accurately. With the holographic methods [1-4], the analysis of tilts in the presence of lateral movements, for instance, is rather complicated and the determination of the adequate plane of localisaticn of the interference fringes is usually not very accurate. These problems do not occur by the application of speckling to the study of three-dimensional movements and in addition no reference wave is needed. The method to be proposed will therefore be a very useful engineering tool.

In the present method two or more practically identical speckle patterns of the optically rough object surface, before and after the tilts, are recorded in the Fourier plane of the object. Illuminating the developed photographic plate with a laser beam yields the Young interference fringes with a spacing inversely proportional to the tilt angle. A brief theory supported by experimental data will be given.
2. Study of the fringes obtained from the speckle patterns recorded in the Fourier plane of the object

We assume that the smallest tilt to be measured induces a shift of the speckles in the Fourier plane at least as large as the smallest speckle dimension, which is inversely porportional to the lateral size of the object field [14-17].

It was shown [5-13] that by recording two identical but shifted speckle patterns by double exposure in the image plane, for instance, Young's fringes are obtained in its Fraunhofer plane, when illuminated

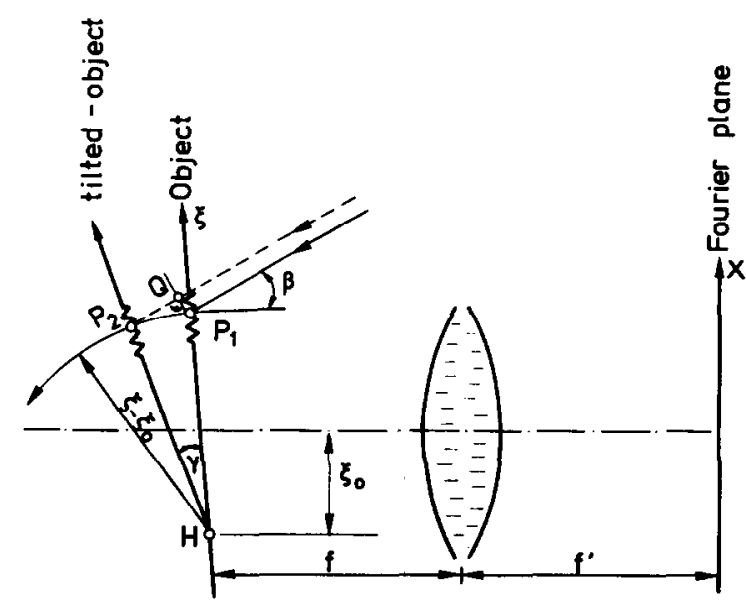

Fig. 1. Arrangement to record the speckles in the Fourier plane. 
with coherent light. Their spacing is inversely proportional to the lateral shift of the speckle pattern. In deriving the appropriate equations use has been made of the fact, that a lateral shift in the object- or image plane, respectively, yields a phase factor proportional to the shift in the Fourier domain, which is commonly realized in the rear focal plane of a lens with focal length $f_{1}$. The novel feature of the experiments reported here is, that the shift in the plane where the speckling is recorded is due to a tilt in another plane. A tilt by an angle $\gamma$ (fig. 1) changes the original wavefront

$A(\xi) \exp [(2 \pi \mathrm{i} \xi \sin \beta) / \lambda]$

into

$A(\xi) \exp [(2 \pi \mathrm{i} \xi \sin \beta) / \lambda] \exp [2 \pi \mathrm{i} \xi \Delta / \lambda]$

( $\lambda=$ wavelength in vacuum). But this leads to a lateral shift of the original speckle pattern

$a[x-(\sin \beta) / \lambda]$

in the Fourier plane, namely

$a[x-(\sin \beta) / \lambda-\Delta / \lambda]$.

After photographic development of the doubly exposed plate with

$|a[x-(\sin \beta) / \lambda]|^{2}+|a[x-(\sin \beta) / \lambda-\Delta / \lambda]|^{2}$,

another Fourier transform in coherent light is performed yielding Young's fringes. An additional lateral shift of the object introduces a linear phase change proportional to it in the Fourier plane of the object and has therefore no consequence since we record the intensity only.

The shift $\Delta$ needs now to be found for an oblique incident plane wave, which is very convenient to simplify the experiments. Contrary to the analysis of inplane movements, where the speckling is recorded in the image plane, the fringe spacing obtained in the proposed way is a function of the incident angle $\beta$ of the illumination (fig. 6). Furthermore the one-dimensional notation is chosen to simplify the writing. If the center of rotation is at $\mathrm{H}$ as shown in fig. 1, the iden- tical point $P_{1}$ on the rough surface moves to $P_{2}$ introducing an optical path change in to the incident oblique wave of

$$
\begin{aligned}
& {\left[\mathrm{QP}_{2}\right]=2\left(\xi-\xi_{0}\right) \sin \frac{1}{2} \gamma \cos \left(\beta-\frac{1}{2} \gamma\right)} \\
& \quad \approx\left(\xi-\xi_{0}\right) \gamma \cos \beta
\end{aligned}
$$

for small tilts $\gamma$ and by assuming the refractive index in air to be 1 . The total change of the original identical wave front

$$
A(\xi) \exp [(2 \pi \mathrm{i} \xi \sin \beta) / \lambda]
$$

is therefore

$$
\begin{gathered}
A(\xi) \exp [(2 \pi \mathrm{i} \xi \sin \beta) / \lambda] \exp [2 \pi \mathrm{i} \xi \gamma / \lambda] \\
\times \exp \left[\left\{2 \pi \mathrm{i}\left(\xi-\xi_{0}\right) \gamma \cos \beta\right\} / \lambda\right]
\end{gathered}
$$

The original identical speckle patterns

$a[x-(\sin \beta) / \lambda]$

in the Fourier plane is therefore shifted and can be written as

$$
\begin{gathered}
a[x-(\sin \beta) / \lambda-\gamma(1+\cos \beta) / \lambda \\
\times \exp \left[\left(2 \pi \mathrm{i} \xi_{0} \gamma \cos \beta\right) / \lambda\right] .
\end{gathered}
$$

The recorded intensity $i(x)$ is by neglecting constants

$$
\begin{aligned}
i(x) & \approx|a[x-(\sin \beta) / \lambda]|^{2}+\mid a[x-(\sin \beta) / \lambda- \\
& -\gamma(1+\cos \beta)]\left.\right|^{2} .
\end{aligned}
$$

From the width $\Delta \xi^{\prime}$ of the cosine fringes obtained in the Fourier plane of the coherently illuminated developed photographic plate the tilt in the object is found to be

$\gamma=\frac{\lambda f_{1}}{f(1+\cos \beta) \Delta \xi^{\prime}}$,

where $f$ and $f_{1}$ are the focal lengths of the lens systems used to perform the Fourier transform from the object and photographic plate respectively. In addition the cosine fringes are multiplied by the square of the autocorrelation of the object field. 


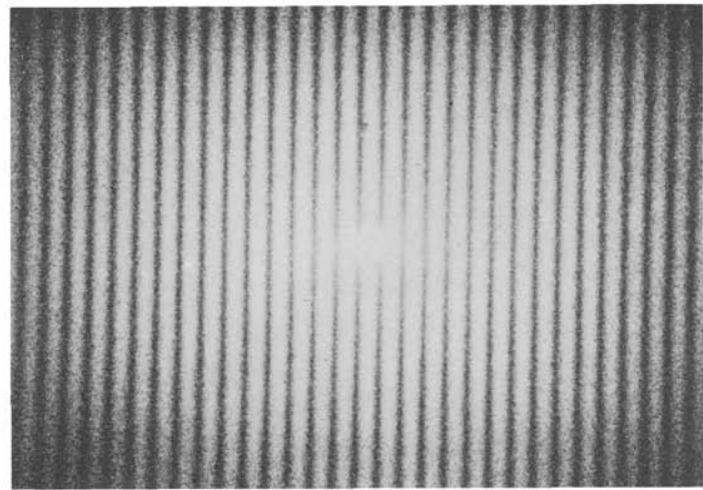

(a)

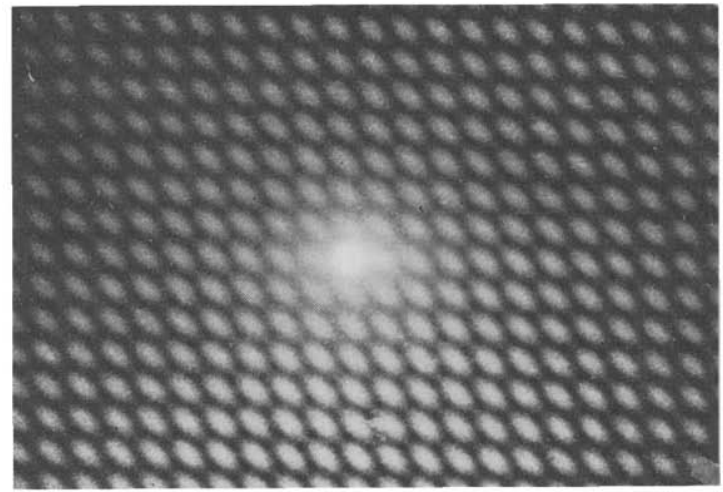

(b)

Fig. 2. Photographs of the fringes obtained from an Al-plate $\left(\beta=45^{\circ}\right)$ for (a) doubly exposed speckle pattern with a tilt of $\gamma_{\xi}=90^{\prime \prime}$ introduced between the exposures; (b) two doubly exposed speckle pattern with tilts of $\gamma_{\xi}=60^{\prime \prime}$ in the plane of $\beta$ and $\gamma_{\eta}=60^{\prime \prime}$ perpendicular to it for the third exposure.

Some experimental results obtained are displayed in fig. 2 for tilts introduced in the plane of $\beta$ (fig. 1) and perpendicular to it and a close agreement between the experimental results and the theory was achieved.

The approach was then extended to multiply exposed speckle patterns where $N$ tilts were introduced between the $(N+1)$ exposures (fig. 3 ).

\section{Double exposure with two beam illumination}

In a further brief study the analysis for two illuminating plane waves incident at the angles $\beta_{1}$ and $\beta_{2}$ to the perpendicular of the object will be considered. The Fourier transforms obtained are similar to those of the previous section but for two beam illumination, namely before the tilt

$\bar{a}_{0}(x)=a_{1}\left[x-\left(\sin \beta_{1}\right) / \lambda\right]+a_{2}\left[x-\left(\sin \beta_{2}\right) / \lambda\right]$

and after the tilt

$$
\begin{aligned}
& \bar{a}_{1}(x)=a_{1}\left[\dot{x}-\left(\sin \beta_{1}\right) / \lambda-\left(1+\cos \beta_{1}\right) \gamma / \lambda\right] \\
& \quad \times \exp \left[\left(2 \pi \mathrm{i} \xi_{0} \gamma \cos \beta_{1}\right) / \lambda\right] \\
&+a_{2}\left[x-\left(\sin \beta_{2}\right) / \lambda-\left(1+\cos \beta_{2}\right) \gamma / \lambda\right] \\
& \quad \times \exp \left[\left(2 \pi \mathrm{i} \xi_{0} \gamma \cos \beta_{2}\right) / \lambda\right] .
\end{aligned}
$$

The intensity $\left|\overline{a_{0}}(x)\right|^{2}+\left|\overline{a_{1}}(x)\right|^{2}$ is recorded on a photographic plate which is developed and then illuminated with coherent light.

In the Fourier plane of the plate we obtain by neglecting all the constants

$A_{1}^{\prime}\left(u^{\prime}\right)=\int\left\{\left|\overline{a_{0}}(x)\right|^{2}+\left|\overline{a_{1}}(x)\right|^{2}\right\} \exp \left[2 \pi \mathrm{i} u^{\prime} x\right] \mathrm{d} x$,

where $u^{\prime}=\xi^{\prime} f / f_{1}\left(\xi^{\prime}=\right.$ rectangular coordinate in the Fourier plane; $f, f$ focal lengths). Expanding the above experession yields cross terms such as

$$
\begin{aligned}
\int a_{1}\left[x-\left(\sin \beta_{1}\right) / \lambda-\left(1+\cos \beta_{1}\right) \gamma / \lambda\right] \\
\quad \times a_{2}^{*}\left[x-\left(\sin \beta_{2}\right) / \lambda-\left(1+\cos \beta_{2}\right) \gamma / \lambda\right] \exp \left[2 \pi \mathrm{i} u^{\prime} x\right] \mathrm{d} x
\end{aligned}
$$

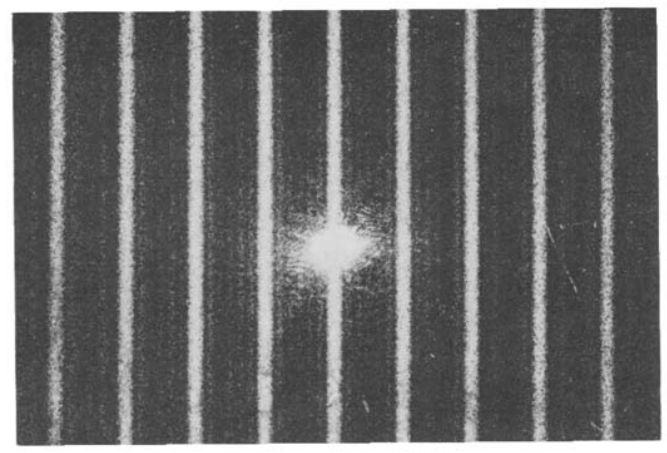

Fig. 3. Photograph of the fringes obtained from a multiple exposed speckle pattern recorded in the Fourier plane of a Cu-plate with 6 equal tilts of $\gamma_{\xi}=30^{\prime \prime}$ between the 7 exposures $\left(\beta=45^{\circ}\right)$. 


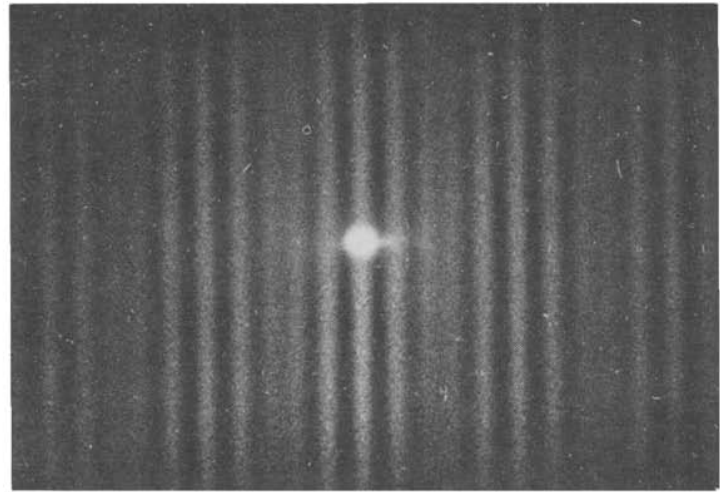

(a)

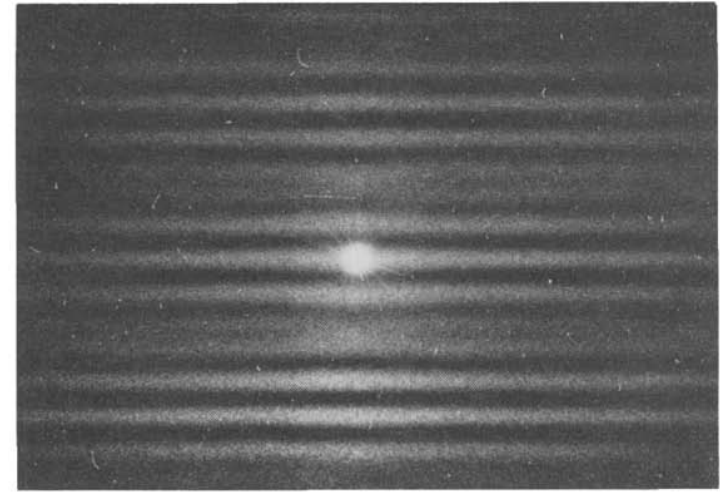

(b)

Fig. 4. Photographs of the fringes obtained by a ground glass as the object and two illuminating beams with unequal angles of incidence $\beta_{1}=30^{\circ}, \beta_{2}=55^{\circ}$ but in the plane of fig. 1.(a) for doubly exposed speckle pattern with a tilt of $\gamma_{\xi}=60^{\prime \prime}\left(\gamma_{\xi} \equiv \gamma\right.$ in fig. 1);

(b) for doubly exposed speckle patterns with a tilt of $\gamma_{\eta}=60^{\prime \prime}$ perpendicular to $\gamma_{\xi}$.

They can, however, be neglected since we integrate over a rapidly varying phase.

The terms making the most significant contributions are

$$
\begin{aligned}
& \int\left\{\left|a_{1}\left[x-\left(\sin \beta_{1}\right) / \lambda\right]\right|^{2}+\left|a_{2}\left[x-\left(\sin \beta_{2}\right) / \lambda\right]\right|^{2}\right. \\
& +\left|a_{1}\left[x-\left(\sin \beta_{1}\right) / \lambda-\left(1+\cos \beta_{1}\right) \gamma / \lambda\right]\right|^{2} \\
& \left.\quad+\left|a_{2}\left[x-\left(\sin \beta_{2}\right) / \lambda-\left(1+\cos \beta_{2}\right) \gamma / \lambda\right]\right|^{2}\right\} \\
& \quad \times \exp \left[2 \pi i u^{\prime} x\right] \mathrm{d} x
\end{aligned}
$$

and the significant contribution to the intensity of the above expression leads to

$$
\begin{aligned}
& I_{1}^{\prime}\left(u^{\prime}\right) \approx 4\left|A_{0}^{\prime}\left(u^{\prime}\right) \otimes A_{0}^{\prime *}\left(-u^{\prime}\right)\right|^{2} \\
& \quad \times\left\{1+\left[\cos \left(2 \pi u^{\prime}\right) \gamma / \lambda\right]\left[1+\left(\cos \beta_{1}+\cos \beta_{2}\right) / 2\right]\right. \\
& \left.\quad \times\left[\cos \left(2 \pi u^{\prime}\right) \gamma / \lambda\right]\left(\cos \beta_{1}-\cos \beta_{2}\right) / 2\right\}
\end{aligned}
$$

where $A_{0}^{\prime}\left(u^{\prime}\right)$ is the Fourier transform of $a(x)$ and $\otimes$ indicates convolution. The cosine fringes in the above expression are therefore modulated by

$\left[\cos \left(2 \pi u^{\prime}\right) \gamma / \lambda\right]\left(\cos \beta_{1}-\cos \beta_{2}\right) / 2$.

For $\beta_{1}= \pm \beta_{2}$ the same results as for one beam illumination are obtained.

There is a close agreement between the theoretical results and the experiments. In fig. 4 we see the ex- perimental results for a two beam illumination of the object and with the speckling recorded in the Fourier plane for $\beta_{1} \neq \beta_{2}$. Tilts were introduced in two directions perpendicular to each other. The modulation of the fringe pattern is clearly visible. The experimental details will be given in the next section. From expression (7) the tilt can be calculated from the separation of the interference fringes $\Delta \xi^{\prime}$.

\section{Experimental procedure}

For our applications a $\mathrm{He}-\mathrm{Ne}$ laser of $18 \mathrm{~mW}$ power and a wavelangth of $\lambda=632,8 \mathrm{~nm}$ was used as a light source. For convenience, the Agfa-Gevaert Scientia 8E75 plates were chosen for the registration of the speckles. Exposure times of a few seconds were necessary. No special care needed to be taken in order to obtain fringes of good contrast. A jig was designed to introduce known tilts into the object illuminated by one or two plane waves. For comparison, the tilts introduced were measured very accurately by autocollimation. It should be noted that the axis of rotation need not coincide with the object surface under test. The additional lateral shift introduced in this way had practically no effects on the tilt fringes observed. The developed photographic plate was illuminated by a converging spherical wave from the $\mathrm{He}-\mathrm{Ne}$ laser and the fringes displayed and photographed in the focal plane. The bright spot in the center is due to the undiffracted light. Fringes could also be seen in white light. 


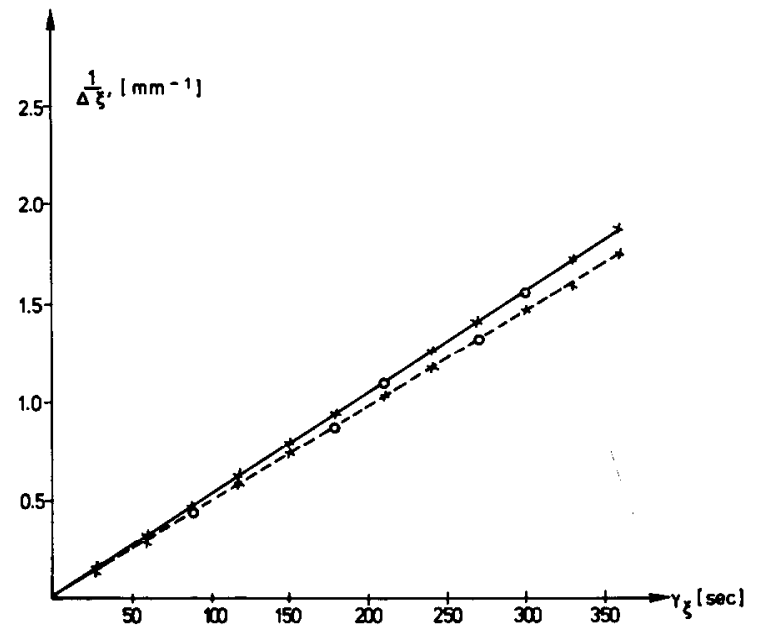

Fig. 5. Results of the experiments where the inverse fringe spacing $1 / \Delta \xi^{\prime}$ is plotted against the measured tilts introduced, theoretical results are drawn - - for $\beta=50^{\circ}$ and $\beta_{1}=30^{\circ}, \beta_{2}=55^{\circ} .0$ indicates the measurements with an additional lateral shift of $40 \mu \mathrm{m}$.

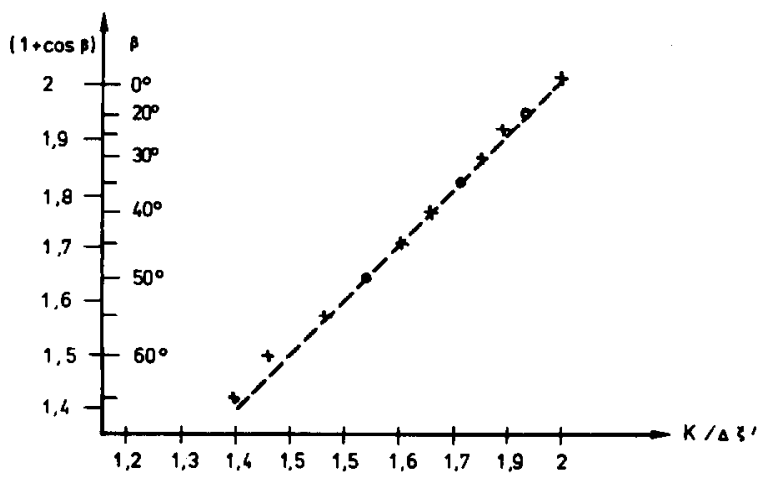

Fig. 6. A typical result of the experiments where the fringe spacing was measured for a given tilt of $\gamma_{\xi}=60^{\prime \prime}$ where $K / \Delta \xi^{\prime}$ is plotted on the abscissa $\left(K=f_{1} \lambda / f \gamma_{\xi}\right)$ and $1+\cos \beta$ on the ordinate for different angles of incidence (points marked by 0 corresponds to tilts $\left.\gamma_{\eta}=60^{\prime \prime}\right)\left(\gamma_{\xi} \perp \gamma_{\eta}\right)$.

Typical results are shown on the photographs where the doubly or multiply exposed speckle patterns were recorded in the Fourier plane of the object. Fringes of good contrast were obtained (fig. 2a) from a single tilt $\left(\gamma_{\xi} \equiv \gamma\right.$ in fig. 1) and equidistant fringes perpendicular to each other from tilts $\gamma_{\xi}$ and $\gamma_{\eta}$ introduced in turn (fig. 2b) $\left(\gamma_{\xi} \perp \gamma_{\eta}\right)$.

A familiar picture is obtained by $N+1$ multiple exposures with $N$ equal tilts between them as from a set of $N+1$ identical equidistant pinholes of equal trans- mission. In between the main maxima, there occur $N-1$ almost equally spaced intermediate maxima considerably weaker than the main ones [13]. The multiple beam fringes become sharper when the number of equal tilts is increased and at the same time the number of intermediate maxima increases. A typical example is shown in fig. 3 , where 7 exposures were introduced with 6 equal tilts between them.

Applying two plane waves to illuminate the object at different angles leads to the additional modulation of the interference fringes due to the tilt as shown in fig. 4 (where the tilt angles $\gamma_{\xi} \perp \gamma_{\eta}$ ).

A vey good linear relationship was obtained between the inverse of the fringe spacing and the tilt angle introduced (fig. 5) (for a given angle of incidence). In fig. 6 $(1+\cos \beta)$ is plotted as function of $K / \Delta \xi^{\prime}$ for different incidence angles keeping $\gamma$ constant $\left(60^{\prime \prime}\right)$. Although the equipment for this experiment.was not very sophisticated, an agreement of better than 4 percent with the theory was achieved for the angles investigated, but a further study may be useful. The results obtained did not seem to depend much on the roughness of the object, but its scale should be fine enough to spread the light over an adequate area in the Fourier plane. We observed a slight decrease in contrast for a coarse surface roughness (very coarse ground glass). If the light is multiply scattered, for example by translucend surfaces, the speckle pattern becomes non-correlated and no fringes are visible.

\section{Acknowledgements}

The author would like to thank the Director of the Institute of Applied Physics and the Department of Industrial Research at the ETH-Z, Professor Dr. E. Baumann, for the support of the work. Thanks are futher due to Professor Th. Huang for reading the manuscript and A. Pochelon for helping with some of the experiments for the photographs of this paper.

\section{References}

[1] C. Froehly, J. Monneret, J. Pasteur and J.Ch. Vienot, Opt. Acta 16 (1969) 343.

[2] J. Monneret, Proc. Intern. Symp. on Holography, Besançon (1970). 
[3] J. Tsujichi, Proc. Intern. Symp. on Holography, Besançon (1970).

[4] J.W.C. Gates, Opt. Technol. 2 (1969) 247.

[5] J.A. Leendertz, J. Phys. E.: Sci. Instr. 3 (1970) 214.

[6] S. Debrus, M. Francon and M. May, Proc. of CIO Conference (1969).

[7] J.N. Butters and J.A. Leendertz, J. Phys. E.: Sci. Instr. 4 (1971) 277.

[8] E. Archbold, J.M. Burch and A.E. Ennos, Opt. Acta 17 (1970 883.

[9] H.J. Tiziani, Opt. Acta 18 (1971) 891.
[10] H.J. Tiziani, Optik 34 (1972) 442.

[11] U. Köpf, Optik 33 (1971) 517.

[12] D. Joyeux and S. Lowenthal, Opt. Commun. 4 (1971) 108.

[13] J.M. Burch and J.M.J. Tokarski, Opt. Acta 15 (1968) 101

[14] L.I. Goldfischer, J. Opt. Soc. Am. 55 (1965) 247.

[15] H.J. Tiziani, Thesis, London University (1967).

[16] H.H. Hopkins and H.J. Tiziani, Proc. Intern. Symp. on Holography, Besançon (1970).

[17] J.C. Dainty, Opt. Acta 17 (1970) 761. 